九州大学学術情報リポジトリ

Kyushu University Institutional Repository

\title{
A LOCATION SHIFT PROBLEM IN NONPARAMETRIC DENSITY ESTIMATION
}

Takeuchi, Hiroyuki

Department of Administration Engineering, Keio University

https://doi.org/10.5109/13432

出版情報: Bulletin of informatics and cybernetics. 25 (3/4), pp.195-212, 1993-03. Research Association of Statistical Sciences

バージョン：

権利関係 : 


\title{
A LOCATION SHIFT PROBLEM IN NONPARAMETRIC DENSITY ESTIMATION
}

By

\author{
Hiroyuki Takeuchi*
}

\begin{abstract}
Let $X_{1}, X_{2}, \ldots, X_{n}$ be i.i.d. random variables having a probability density function $f(x)$ and $f_{n}(x)$ be a nonparametric density estimator of $f(x)$. Wc investigate the property of a location shift random variable $a_{n}$ which minimizes integrated squared error $\operatorname{ISE}_{n}(a)$ :

$$
\operatorname{ISE}_{n}(a)=\int_{-\infty}^{\infty}\left|f_{n}(x)-f(x-a)\right|^{2} d x .
$$

The asymptotic normality and the order of strong convergence of the r.v. $a_{n}$ and those of $\operatorname{ISE}_{n}\left(a_{n}\right)$ are studied. We also give some numerical examples and some simulations which show the effectiveness of using the $a_{n}$ when one estimates $f(x)$ by $f_{n}(x)$.
\end{abstract}

\section{Introduction}

Let

$$
X_{1}, X_{2}, \ldots, X_{n}
$$

be independently and identically distributed random variables with a common distribution function $F(x)$ whose density is $f(x)$. We define $f_{n l}(x)$ :

$$
f_{n}(x)=\frac{1}{n} \sum_{j=1}^{n} K_{n}\left(x-X_{j}\right)
$$

as a nonparametric density estimator for $f(x)$. The estimator $f_{n}(x)$ has been so widely studied by many authors, see, for example Izenman [11] or Prakasa Rao [14]. In this paper we shall investigate the asymptotic property of the location shift r.v. $a_{n}$ which minimizes the integrated squared error $\operatorname{ISE}_{n}(a)$ :

$$
\operatorname{ISE}_{n}(a)=\int_{-\infty}^{\infty}\left|f_{n}(x)-f(x-a)\right|^{2} d x
$$

Blackman [2] considered this problem for the empirical distribution function and he got the asymptotic distribution of $\sqrt{n} a_{n}$. In Härdle [9], some estimators for the shift parameter are cited for the robust estimation of nonparametric regression function.

\footnotetext{
* Department of Administration Engineering, Keio University, 3-14-1 Hiyoshi, Kohoku-ku, Yokohama 223. Japan.
} 
Scott [16] defined averaged shifted histograms but it seems that there is little connection with our work.

In Section 2, we shall show the weak convergence of the $a_{n}$ using Heathcote's idea [10]. Since our situation is nonparametric, so the proof is slightly more complicated than his. We show that the asymptotic variance of $\sqrt{n} a_{n}$ is inherent in the underlying distribution. The asymptotic normality of $\operatorname{ISE}_{n}(a)-\operatorname{ISE}_{n}(0)$ is also proved to show the range of the interval $\left\{a: \operatorname{ISE}_{n}(a)-\operatorname{ISE}_{n}(0)<0\right\}$. In Section 3 , the order of strong convergence of the $a_{n}$ is considered for the kernel-type density estimator. In that section, the empirical characteristic function based on (1.1) reveals us a powerful tool to evaluate the order. Especially it is proved that if $\hat{a}_{n}$ is any estimator of the location shift r.v. $a_{n}$ and $h_{n}$ is the window width, then $\operatorname{ISE}_{n}\left(\hat{a}_{n}\right)-\operatorname{ISE}_{n}(0)$ can not converge to 0 slower than $O\left(\left(\frac{\log \log n}{n}\right)^{\frac{1}{4}\left(1-\frac{1}{\mu-1}\right)}\right)$ or $O\left(h_{n}^{1 / 2}\right)$, for some $\mu>2$, according to certain conditions. A simulation was conducted in Section 4 to estimate the relative efficiency of the estimation with respect to the location shift r.v. $a_{n}$.

\section{Weak Convergence of the Location Shift Random Variable $a_{n}$ and $\operatorname{ISE}_{n}(a)$}

In this section we shall consider a class of the density estimator $\left\{f_{n}(x), n \in N\right\}$ that can be written in the next form

$$
f_{n}(x)=\frac{1}{n} \sum_{j=1}^{n} K_{n}\left(x-X_{j}\right)
$$

where $\left\{K_{n}, n \in N\right\}$ is a sequence of "kernel" functions. Many types of density estimators are contained in this class, for example, kernel estimators, trigonometric series estimators, orthogonal polynomial estimators, Fourier transform estimators and histogram estimators (Hall [7]). We can write the Fourier transform of (2.1) as,

$$
\varphi_{f_{n}}(t)=\varphi_{K_{n}}(t) \cdot c_{n}(t)
$$

where $c_{n}(t)$ is called the empirical characteristic function (e.c.f.), based on (1.1), defined by

$$
c_{n}(t)=\frac{1}{n} \sum_{j=1}^{n} e^{i X_{j} t}
$$

It is obvious that $c_{n}(t)$ converges to $\varphi_{f}(t)$, the characteristic function of the $f(x)$, with probability one for each $t \in \boldsymbol{R}$ (Lukacs [12]). The property of the e.c.f. has been investigated by many authors, for example Csörgö [5], Marcus [13] and Feuerverger \& Mureika [6].

We assume that the true probability density function (p.d.f.) $f(x)$ satisfies (2.2).

$$
\int_{-\infty}^{\infty} t^{2}\left|\varphi_{f}(t)\right| d t<\infty .
$$

And we suppose following conditions for the kernel.

$$
K_{n}(y)=K_{n}(-y) \text {, for all } n \in N,
$$




$$
\begin{gathered}
\sup _{n \in N} \int_{-\infty}^{\infty}\left|K_{n}(y)\right| d y<\infty, \\
K_{n}(y) \in L^{2}(\boldsymbol{R}) . \\
\lim _{n \rightarrow \infty} \varphi_{K_{n}}(t)=1, \text { for each } t \in \boldsymbol{R} .
\end{gathered}
$$

We use the symbol $\operatorname{Re}[z]$ and $\operatorname{Im}[z]$ as the real and the imaginary part of $z \in C$, respectively. The complex number $\bar{z}$ denotes the conjugate of $z$. And the symbols $E$ and $\operatorname{Var}$ denote the expectation and the variance, respectively, with corresponding probability measure $P$ or the measure generated by $F(\cdot)$. Furthermore a.s. $\stackrel{p}{\longrightarrow}$ and $\stackrel{S}{\longrightarrow}$ denotes the convergence with probability one, in probability and in distribution, respectively.

To state Theorem 2.2 and 2.3 , the main results of this section, we need the following lemmas.

LEMMA 2.1.

If

$$
\int_{-\infty}^{\infty}\left|t \varphi_{f}(t)\right|\left|\varphi_{K_{n}}(t)-1\right| d t=o\left(n^{-1 / 2}\right)
$$

for large $n$, then we have

$$
\begin{aligned}
& \frac{1}{\sqrt{n}} \sum_{j=1}^{n} \operatorname{Im}\left[\int_{-\infty}^{\infty} t e^{i X_{j t}} \cdot \varphi_{K_{n}}(t) \cdot \overline{\varphi_{f}(t)} \mathrm{dt}\right] \\
& \stackrel{\mathscr{s}^{\prime}}{\longrightarrow} N\left(0, E\left|\operatorname{Im}\left[\int_{-\infty}^{\infty} t e^{i X_{1 t}} \cdot \overline{\varphi_{f}(t)} \mathrm{dt}\right]\right|^{2}\right), \text { as } n \rightarrow \infty .
\end{aligned}
$$

Proof. From theorem 4.1 in Billingsley [1], we may prove (2.9) and (2.10) to show (2.8).

$$
\begin{aligned}
& \frac{1}{\sqrt{n}} \sum_{j=1}^{n} \lim _{n \rightarrow \infty} \Psi_{n}\left(X_{j}\right) \stackrel{g}{\longrightarrow} N\left(0, E\left|\operatorname{Im}\left[\int_{-\infty}^{\infty} t e^{i X_{1} t} \cdot \overline{\varphi_{f}(t)} d t\right]\right|^{2}\right), \\
& \left|\frac{1}{\sqrt{n}} \sum_{j=1}^{n} \Psi_{n}\left(X_{j}\right)-\frac{1}{\sqrt{n}} \sum_{j=1}^{n} \lim _{n \rightarrow \infty} \Psi_{n}\left(X_{j}\right)\right| \stackrel{p}{\longrightarrow} 0 .
\end{aligned}
$$

as $n \rightarrow \infty$, where $\Psi_{n}(X)$ is defined by

$$
\Psi_{n}(X)=\operatorname{Im}\left[\int_{-\infty}^{\infty} t e^{i X t} \cdot \varphi_{K_{n}}(t) \cdot \overline{\varphi_{f}(t)} d t\right]
$$

By (2.2), (2.4) and (2.6), we can use dominated convergence theorem such as

$$
\lim _{\mathbf{n} \rightarrow \infty} \Psi_{n}\left(X_{j}\right)=\operatorname{Im}\left[\int_{-\infty}^{\infty} t e^{i X_{j} t} \cdot \overline{\varphi_{f}(t)} d t\right] .
$$

The sequence of the right hand side of (2.12), suffixed by $j$, is i.i.d. random variables which have finite second moment by (2.2). The expectation of (2.12) is 0 for all $j$ from 
(2.3), so by virtue of the central limit theorem, we have

$$
\frac{1}{\sqrt{n}} \sum_{j=1}^{n} \lim _{n \rightarrow \infty} \Psi_{n}\left(X_{j}\right) \stackrel{\leftrightarrow}{\longrightarrow} N\left(0, \operatorname{Var}\left[\lim _{n \rightarrow \infty} \Psi_{n}\left(X_{1}\right)\right]\right),
$$

as $n \rightarrow \infty$. Thus we have (2.9) immediately. Then by $(2.7),(2.10)$ is proved in the following way.

$$
\begin{aligned}
& \frac{1}{\sqrt{n}} \sum_{j=1}^{n}\left|\Psi_{n}\left(X_{j}\right)-\lim _{n \rightarrow \infty} \Psi_{n}\left(X_{j}\right)\right| \\
\leq & \frac{1}{\sqrt{n}} \sum_{j=1}^{n}\left|\int_{-\infty}^{\infty} t e^{i X_{j} t}\left(\varphi_{K_{n}}(t)-1\right) \cdot \overline{\varphi_{f}(t)} d t\right| \\
\leq & \sqrt{n} \int_{-\infty}^{\infty}\left|t \varphi_{f}(t)\right|\left|\varphi_{K_{n}}(t)-1\right| \mathrm{dt} \\
\rightarrow & 0, \text { a.s. }
\end{aligned}
$$

Hence we get (2.8).

The condition (2.7) may seem to be technical, but it is not so strong. In fact next proposition can be stated in the kernel-type density estimation case.

Proposition 2.1.

Suppose that the density estimator is a kernel-type;

$$
f_{n}(x)=\frac{1}{n h_{n}} \sum_{j=1}^{n} K\left(\frac{x-X_{j}}{h_{n}}\right) .
$$

where $h_{n}$ is called the window width satisfying $\lim _{n \rightarrow \infty} h_{n}=0$. And assume that the kernel is standard normal and that the characteristic function of true p.d.f. satisfies

$$
\left|\varphi_{f}(t)\right| \leq A e^{-\rho|t|}
$$

for some $A, \rho>0$ that are independent of $t$. If the order of the window width is

$$
h_{n}=o\left(n^{-\frac{1}{4}-t}\right)
$$

then (2.7) is satisfied for any $\varepsilon>0$.

Proof. Since by assumption, for any fixed $\varepsilon>0$ we can choose $m>\frac{1}{2 \varepsilon}$ such that

$$
\begin{aligned}
\int_{n^{\varepsilon}}^{\infty}\left|t \varphi_{f}(t)\right| d t & \leq \frac{A}{\rho^{2}}\left(1+\rho n^{\varepsilon}\right) e^{-\rho n^{t}} \\
& =o\left(n^{-m \varepsilon}\right) \\
& =o(1) .
\end{aligned}
$$

And then we have 


$$
\begin{aligned}
& \sqrt{n} \int_{-\infty}^{\infty}\left|t \varphi_{f}(t)\right|\left|\varphi_{K}\left(h_{n} t\right)-1\right| d t \\
\leq & \sqrt{n} \sup _{|t| \leq n^{*}}\left|\exp \left(-\frac{1}{2} h_{n}^{2} t^{2}\right)-1\right| \int_{-\infty}^{\infty}\left|t \varphi_{f}(t)\right| d t \\
& \quad+2 \sqrt{n}\left(\int_{n^{*}}^{\infty}\left|t \varphi_{f}(t)\right| d t+\int_{-\infty}^{-n^{\varepsilon}}\left|t \varphi_{f}(t)\right| d t\right) \\
= & O\left(h_{n}^{2} \cdot n^{\frac{1}{2}+2 \varepsilon}\right)+o\left(n^{\frac{1}{2}-m \varepsilon}\right) \\
= & o(1)
\end{aligned}
$$

by $(2.15)$.

The class of the characteristic function satisfying (2.14) contains wide variety of p.d.f. The form of (2.14), which was used in Watson and Leadbetter [18], will also appear in Section 3.

We shall evaluate the order of convergence of $a_{n}$ to 0 in Section 3 under some conditions, but we can prove following theorem without any technical conditions.

THEOREM 2.1. The location shift random variable $a_{n}$ converges to 0 with probability one.

Proof. By dominated convergence theorem, we have

$$
\begin{aligned}
& \pi\left\{\operatorname{ISE}_{n}(a)-\operatorname{ISE}_{n}(0)\right\} \\
= & \operatorname{Re}\left[\int_{-\infty}^{\infty}\left(1-e^{-i a t}\right) \varphi_{K_{n}}(t) \cdot c_{n}(t) \cdot \overline{\varphi_{f}(t)} d t\right] \\
\rightarrow & \int_{-\infty}^{\infty}\{1-\cos (a t)\}\left|\varphi_{f}(t)\right|^{2} d t, \text { a.s. }
\end{aligned}
$$

as $n \rightarrow \infty$, for each $a \in R$. This means for any $a \in \boldsymbol{R}$,

$$
\operatorname{ISE}_{n}(a)-\operatorname{ISE}_{n}(0) \geq 0 \text {, a.s. }
$$

for sufficiently large $n$. $\operatorname{ISE}_{n}(a)$ is differentiable about $a \in \boldsymbol{R}$, so there exists a solution $a_{n}$ of the equation

$$
\frac{d}{d a} \operatorname{ISE}_{n}(a)=0
$$

that converges to 0 with probability one. To show that the limit of the $a_{n}$ is 0 only, we shall give a necessary and sufficient conditions for an identifiability condition with respect to $f(x)$ when we consider a location shift parameter for $f(x)$. The following (i), (ii) and (iii) are equivalent

(i) $a=0$

(ii) $\int_{-\infty}^{\infty}\left(1-e^{i a t}\right)\left|\varphi_{f}(t)\right|^{2} d t=0$

(iii) $f(x)=f(x-a)$, almost everywhere in $x \in \boldsymbol{R}$.

(i) to (iii) is obvious. (iii) to (ii) is as follows. 


$$
\int_{-\infty}^{\infty}\left(1-e^{i a t}\right)\left|\varphi_{f}(t)\right|^{2} d t=2 \pi\left(\int_{-\infty}^{\infty}|f(x)|^{2} d x-\int_{-\infty}^{\infty} f(x-a) f(x) d x\right)
$$

by Parseval's relation. Finally we shall show (ii) to (i). If $a \neq 0$ there exists a $\delta>0$ such that if $t \in(0, \delta]$ then both of $1-\cos ($ at $)$ and $\left|\varphi_{f}(t)\right|$ are positive by their continuity. So we have

$$
\begin{aligned}
\operatorname{Re}\left[\int_{-\infty}^{\infty}\left(1-e^{i a t}\right)\left|\varphi_{f}(t)\right|^{2} d t\right] & \geq \int_{t \in(0, \delta]}\{1-\cos (a t)\}\left|\varphi_{f}(t)\right|^{2} d t \\
& >0
\end{aligned}
$$

This contradicts (ii), and hence we have (ii) to (i). By the assertion above, the right hand side of (2.16) is 0 if and only if $a=0$. This completes the proof of the theorem.

We need following lemma to prove Lemma 2.3.

Lemma 2.2 Let $(\Omega, \mathscr{F}, P)$ be a probability space and $\left\{X_{l}, X_{m, n}: l, m, n \in N\right\}$ be a sequence of random variables defined on the $(\Omega, \mathscr{F}, P)$. Suppose that

(i) $X_{1}, X_{2}, \ldots$ are independently and identically distributed random variables,

(ii) $E\left|X_{1}\right|<\infty$,

(iii) $\lim _{n \rightarrow \infty} \max _{1 \leq m \leq n}\left|X_{m, n}-X_{m}\right|=0$, a.s.

Then we have

$$
\lim _{n \rightarrow \infty} \frac{1}{n} \sum_{m=1}^{n} X_{m, n}=E X_{1}, \quad \text { a.s. }
$$

Proof. Write

$$
\begin{aligned}
& \left|\frac{1}{n} \sum_{m=1}^{n} X_{m, n}-E X_{1}\right| \\
\leq & \left|\frac{1}{n} \sum_{m=1}^{n} X_{m, n}-\frac{1}{n} \sum_{m=1}^{n} X_{m}\right|+\left|\frac{1}{n} \sum_{m=1}^{n} X_{m}-E X_{1}\right| \\
= & I_{1, n}+I_{2, n},
\end{aligned}
$$

say. From (i) and (ii), we have

$$
\lim _{n \rightarrow \infty} I_{2, n}=0, \quad \text { a.s. }
$$

by Kolmogorov's strong law of large numbers. From (iii), there exists an $\Omega_{0} \in \mathscr{F}$ such that $P\left(\Omega_{0}\right)=1$ and that

$$
{ }^{\forall} \omega \in \Omega_{0},{ }^{\forall} \varepsilon>0,{ }^{\exists} N(\omega): n \geq N(\omega) \Rightarrow \max _{1 \leq m \leq n}\left|X_{m, n}(\omega)-X_{m}(\omega)\right|<\varepsilon .
$$

Hence, then we have

$$
\begin{aligned}
I_{1, n} & \leq \frac{1}{n} \sum_{m=1}^{n}\left|X_{m, n}(\omega)-X_{m}(\omega)\right| \\
& <\varepsilon .
\end{aligned}
$$

This completes the proof. 
Lemma 2.3 .

$$
\begin{gathered}
\frac{1}{n} \sum_{j=1}^{n} \operatorname{Re}\left[\int_{-\infty}^{\infty} t^{2} e^{i\left(X_{j}-\theta a_{n}\right) t} \cdot \varphi_{K_{n}}(t) \cdot \overline{\varphi_{f}(t)} d t\right] \\
\rightarrow \int_{-\infty}^{\infty} t^{2}\left|\varphi_{f}(t)\right|^{2} d t, \quad \text { a.s. }
\end{gathered}
$$

as $n \rightarrow \infty$, uniformly for $\theta \in(0,1)$, where $\theta$ is depending on $f, K_{n}$ and $a_{n}$.

Proof. We define r.v. $Y_{j}$ as

$$
Y_{j}=\operatorname{Re}\left[\int_{-\infty}^{\infty} t^{2} e^{i X_{j} t} \cdot \overline{\varphi_{f}(t)} d t\right]
$$

and $Y_{j, n}$ as

$$
Y_{j, n}=\operatorname{Re}\left[\int_{-\infty}^{\infty} t^{2} e^{i\left(X_{j}-\theta a_{n}\right) t} \cdot \varphi_{K_{n}}(t) \cdot \overline{\varphi_{f}(t)} d t\right]
$$

Note that $Y_{j, n}$ is equivalent to

$$
-\left.\frac{d}{d u} \Psi_{n}\left(X_{j}-u\right)\right|_{u=\theta a_{n}}
$$

where $\Psi_{n}(X)$ is defined by (2.11). We shall show the sequence $\left\{Y_{j}, Y_{j, n}: j, n \in N\right\}$ satisfies the condition (i), (ii) and (iii) of Lemma 2.2. (i) is obvious. (ii) is also clear from (2.2). Next we show (iii).

$$
\begin{aligned}
& \max _{1 \leq j \leq n}\left|Y_{j, n}-Y_{j}\right| \\
\leq & \int_{-\infty}^{\infty} t^{2}\left|\varphi_{f}(t)\right|\left|e^{-i \theta a_{n} t} \cdot \varphi_{K_{n}}(t)-1\right| d t \\
\rightarrow & 0, \quad \text { a.s. }
\end{aligned}
$$

as $n \rightarrow \infty$ by (2.4). (2.6) and Theorem 2.1 with applying dominated convergence theorem. Finally we get

$$
E Y_{1}=\int_{-\infty}^{\infty} t^{2}\left|\varphi_{f}(t)\right|^{2} d t
$$

by Fubini's theorem.

Now we can state next theorem.

THEOREM 2.2. Under the condition of Lemma 2.1, we have

$$
\sqrt{n} a_{n} \stackrel{\infty}{\longrightarrow} N\left(0, \frac{E\left|\operatorname{Im}\left[\int_{-\infty}^{\infty} t e^{i X_{1 t}} \cdot \overline{\varphi_{f}(t)} d t\right]\right|^{2}}{\left(\int_{-\infty}^{\infty} t^{2}\left|\varphi_{f}(t)\right|^{2} d t\right)^{2}}\right)
$$

as $n \rightarrow \infty$.

Proof. From (2.2) and (2.5) we have $f_{n}, f \in L^{2}(\boldsymbol{R})$, so (1.2) is equivalent to 


$$
\operatorname{ISE}_{n}(a)=\frac{1}{2 \pi} \int_{-\infty}^{\infty}\left|\varphi_{K_{n}}(t) \cdot c_{n}(t)-e^{i a t} \cdot \varphi_{f}(t)\right|^{2} d t
$$

by Parseval's relation. (2.2) affords us interchange of differentiation and integration such that

$$
\begin{aligned}
& \frac{d}{d a} \operatorname{ISE}_{n}(a) \\
= & -\frac{1}{\pi} \cdot \operatorname{Im}\left[\int_{-\infty}^{\infty} t e^{-i a t} \cdot \varphi_{K_{n}}(t) \cdot c_{n}(t) \cdot \overline{\varphi_{f}(t)} d t\right] .
\end{aligned}
$$

So, with $\Psi_{n}$ defined in $(2.11)$, the problem becomes to find an $a_{n}$ which satisfies the equation:

$$
\sum_{j=1}^{n} \Psi_{n}\left(X_{j}-a\right)=0
$$

Characteristic of $a_{n}$ which satisfies $\sum_{j=1}^{n} \rho\left(X_{j}-a\right)=0$ has been discussed by Huber [11], where $\rho(\cdot)$ is a continuous function not depending on $n$. Let $a_{n}$ be a consistant solution of this equation. Then

$$
\begin{aligned}
0 & =\sum_{j=1}^{n} \Psi_{n}\left(X_{j}-a_{n}\right) \\
& =\sum_{j=1}^{n} \Psi_{n}\left(X_{j}\right)+\left.a_{n} \sum_{j=1}^{n} \frac{d}{d u} \Psi_{n}\left(X_{j}-u\right)\right|_{u=\theta a_{n}}
\end{aligned}
$$

for some $\theta \in(0,1)$ by Taylor's expansion. So we get

$$
\sqrt{n} a_{n}=\frac{-\frac{1}{\sqrt{n}} \sum_{j=1}^{n} \Psi_{n}\left(X_{j}\right)}{\left.\frac{1}{n} \sum_{j=1}^{n} \frac{d}{d u} \Psi_{n}\left(X_{j}-u\right)\right|_{u=\theta a_{n}}}
$$

Hence from Lemma 2.1 and Lemma 2.3, we obtain the conclusion by Slutsky's theorem (Serfling [17]).

We remark that the asymptotic variance of $\sqrt{n} a_{n}$ does not depend on the kernel and it only depends on the true p.d.f., in other words, the asymptotic variance is inherent in the underlying distribution $F$.

Example 1. We exhibit the asymptotic variance in Theorem 2.2 for Cauchy and normal distributions;

$$
\begin{aligned}
& \varphi_{f}(t)=e^{-s|t|}, s>0, \\
& \varphi_{f}(t)=e^{-\sigma^{2} t^{2} / 2}, \sigma>0 .
\end{aligned}
$$

The asymptotic variances are $\frac{5}{2} s^{2}$ and $\frac{8}{3 \sqrt{3}} \sigma^{2}$, respectively.

Hereafter we define $\operatorname{DISE}_{n}(a)$ as 


$$
\operatorname{DISE}_{n}(a)=\operatorname{ISE}_{n}(a)-\operatorname{ISE}_{n}(0)
$$

for simplification.

THEOREM 2.3. Under the condition of Lemma 2.1, we have

$$
\operatorname{limP}_{\mathrm{n} \rightarrow \infty}\left[\sqrt{n} \pi \cdot \operatorname{DISE}_{n}(a)<x\right]=\Phi_{\mu_{a}, \sigma_{a}^{2}}(x)
$$

for each $a \neq 0$, where $\Phi_{\mu_{a}, \sigma_{a}^{2}}(x)$ denotes a normal distribution function with mean $\mu_{a}$ and variance $\sigma_{a}^{2}$;

$$
\begin{gathered}
\mu_{a}=\int_{-\infty}^{\infty}\{1-\cos (a t)\}\left|\varphi_{f}(t)\right|^{2} d t \\
\sigma_{a}^{2}=E\left|\operatorname{Re}\left[\int_{-\infty}^{\infty}\left(1-e^{-i a t}\right) e^{i X_{1} t} \cdot \overline{\varphi_{f}(t)} d t\right]\right|^{2}-\mu_{a}^{2}
\end{gathered}
$$

respectively.

Proof. The proof is almost the same as that of Lemma 2.1, so we shall only show the outline here. We define $\Psi_{n}\left(X_{j}, a\right)$ as

$$
\Psi_{n}\left(X_{j}, a\right)=\operatorname{Re}\left[\int_{-\infty}^{\infty}\left(1-e^{-i a t}\right) e^{i X_{j t}} \cdot \varphi_{K_{n}}(t) \cdot \overline{\varphi_{f}(t)} d t\right]
$$

then from (2.2) and (2.6), we have

$$
\lim _{n \rightarrow \infty} \Psi_{n}\left(X_{j}, a\right)=\operatorname{Re}\left[\int_{-\infty}^{\infty}\left(1-e^{-i a t}\right) e^{i X_{\mu}} \cdot \overline{\varphi_{f}(t)} d t\right],
$$

by dominated convergence theorem. We can show (2.23) and (2.24),

$$
\begin{gathered}
\frac{1}{\sqrt{n}} \sum_{j=1}^{n} \lim _{n \rightarrow \infty} \Psi_{n}\left(X_{j}, a\right) \stackrel{\infty}{\longrightarrow} N\left(\mu_{a}, \sigma_{a}^{2}\right), \\
\left|\frac{1}{\sqrt{n}} \sum_{j=1}^{n} \Psi_{n}\left(X_{j}, a\right)-\frac{1}{\sqrt{n}} \sum_{j=1}^{n} \lim _{n \rightarrow \infty} \Psi_{n}\left(X_{j}, a\right)\right| \stackrel{p}{\longrightarrow} 0,
\end{gathered}
$$

as $n \rightarrow \infty$. By the definition of $\operatorname{ISE}_{n}(a)$, we have thus proved

$$
\sqrt{n} \pi \cdot \operatorname{DISE}_{n}(a)=\frac{1}{\sqrt{n}} \sum_{j=1}^{n} \Psi_{n}\left(X_{j}, a\right) \stackrel{s}{\longrightarrow} N\left(\mu_{a}, \sigma_{a}^{2}\right), \quad \text { as } n \rightarrow \infty
$$

for each $a \neq 0$.

By the Theorem 2.3 we can find the range of the interval of which the parameter $a$ satisfies $\operatorname{DISE}_{n}(a)<0$. $\operatorname{ISE}_{n}(0)$ is thought of as the loss when we do not take the parameter into consideration. Following example illustrates the contents above.

EXAMPLE 2. If $\varphi_{f}(t)=\exp \left(-\frac{1}{2} t^{2}\right)$ then (2.2) is satisfied. In this case we have the asymptotic mean $\mu_{a}$ and the asymptotic variance $\sigma_{a}^{2}$ as follows. 


$$
\begin{aligned}
\mu_{a} & =\sqrt{\pi}\left(1-e^{-a^{2} / 4}\right), \\
\sigma_{a}^{2} & =\pi\left(1-e^{-a^{2} / 4}\right)\left(e^{-a^{2} / 4}+4 / \sqrt{3}-1\right),
\end{aligned}
$$

Obviously the probability $p(a)=\lim _{n \rightarrow \infty} P\left\{\operatorname{DISE}_{n}(a)<0\right\}$ satisfies $p(a)=p(-a)$. Furthermore we remark that $p(a)$ is a continuous function about the parameter $a$, except for the point $a=0$, and $\lim _{a \rightarrow 0} p(a)=0.5$. Table 1 shows an example of the values of $p(a)$.

Table 1. Values of $p(a)$

\begin{tabular}{|c||cccccccccc|}
\hline$a$ & 0 & 0.1 & 0.2 & 0.3 & 0.4 & 0.5 & 0.6 & 0.7 & 0.8 \\
$p(a)$ & 0 & 0.49 & 0.47 & 0.46 & 0.45 & 0.43 & 0.42 & 0.41 & 0.40 \\
\hline
\end{tabular}

\section{Strong Consistency of the Location Shift Random Variable $a_{n}$ and $\operatorname{ISE}_{n}\left(a_{n}\right)$}

In this section we shall evaluate the order of strong convergence of the $a_{n}$, and confine ourselves to consider the kernel-type density estimator which is given by (2.13), where the kernel $K(y)$ satisfies following three conditions,

$$
\begin{gathered}
\forall y \in \boldsymbol{R}, K(y) \geq 0 \text { and } \int_{-\infty}^{\infty} K(y) d y=1, \\
K(y) \in L^{2}(\boldsymbol{R}), \\
\int_{-\infty}^{\infty}|y K(y)| d y<\infty .
\end{gathered}
$$

Hereafter we define the sequence $\left\{T_{n}, n \in N\right\}$ as

$$
T_{n}=\frac{\log \log n}{n} .
$$

To evaluate the convergence order of the $a_{n}$ to 0 , we need following lemma that shows the uniform strong convergence of the e.c.f. process. Note that the lemma is slightly different from Csörgö [5] who proved this first.

Lemma 3.1. For any p.d.f. $f(x)$, we have

$$
\sup _{|t| \leq U_{n}}\left|c_{n}(t)-\varphi_{f}(t)\right|=O\left(U_{n} \cdot T_{n}^{1 / 2}\right), \text { a.s. }
$$

for sufficiently large $n$, where $\left\{U_{n}, n \in N\right\}$ is a positive monotone increasing sequence diverging to infinity.

Proof. Using integration by parts, we have for any fixed $K>0$, 


$$
\begin{aligned}
& \sup _{|t| \leq U_{n}}\left|c_{n}(t)-\varphi_{\mathrm{f}}(t)\right| \\
= & \sup _{|t| \leq U_{n}}\left|\int_{-x}^{\infty} e^{i t x} d\left(F_{n}(x)-F(x)\right)\right| \\
\leq & \sup _{|t| \leq U_{n}}\left|\left[e^{i t x}\left(F_{n}(x)-F(x)\right)\right]_{-K}^{K}-i t \int_{-K}^{K} e^{i t . x}\left(F_{n}(x)-F(x)\right) d x\right| \\
& \quad+\sup _{|t| \leq U_{n}}\left|\int_{x \in[-K, K]^{c}} e^{i t x} d\left(F_{n}(x)-F(x)\right)\right| \\
\leq & \left(4+2 K U_{n}\right) \sup _{-x<x<\infty}\left|F_{n}(x)-F(x)\right|,
\end{aligned}
$$

where $F_{n}(x)$ denotes the empirical distribution function which based on (1.1). By Chung [4], we have

$$
\limsup _{\mathrm{n} \rightarrow \infty} T_{n}^{-1 / 2} \sup _{-\infty<x<x}\left|F_{n}(x)-F(x)\right|=\frac{1}{\sqrt{2}} \text {, a.s. }
$$

so then

$$
\sup _{-x<x<\infty}\left|F_{n}(x)-F(x)\right|=O\left(T_{n}^{1 / 2}\right), \text { a.s. }
$$

From above and (3.5), we have (3.4) for sufficiently large $n$.

Lemma 3.2. Suppose that the true p.d.f. $f(x)$ satisfies the following.

$$
\int_{-\infty}^{\infty}|t|^{m+1}\left|\varphi_{f}(t)\right| d t<\infty
$$

for some nonnegative integer $m$.

(ii) There exists a $\mu>m$ such that

$$
\int_{t \in S_{n}^{c}}|t|^{m}\left|\varphi_{f}(t)\right| d t=o\left(T_{n^{\frac{u-m}{2 \mu-m+1)}}}\right),
$$

where the set $S_{n}$ is given by

$$
S_{n}=\left\{t: t \in\left[-T_{n^{\frac{-1}{2(u-m+1)}}, T_{n^{2(u-m+1)}}}^{\frac{-1}{2}}\right\} .\right.
$$

Then we have

$$
\begin{aligned}
& \sup _{-\infty<a<x}\left|\frac{d^{v}}{d a^{v}} \operatorname{ISE}_{n}(a)-\lim _{n \rightarrow \infty} \frac{d^{v}}{d a^{v}} \operatorname{ISE}_{n}(a)\right| \\
& =O\left(T_{n}^{\frac{u-m}{2(u-m+1)}}\right), \text { a.s. for } h_{n}=o\left(T_{n^{\frac{u-m}{2(u-m+1)}}}\right) \\
& =O\left(h_{n}\right), \quad \text { a.s. for } T_{n}=o\left(h_{n}^{\frac{2(u-m+1)}{\mu-m}}\right)
\end{aligned}
$$

as $n \rightarrow x$, for $v=0,1, \ldots, m$.

Proof. It is easy to check that 


$$
\int_{-\infty}^{\infty}|t|^{m_{1}}\left|\varphi_{f}(t)\right| d t<x \text { implies } \int_{-\infty}^{\infty}|t|^{m_{2}}\left|\varphi_{f}(t)\right| d t<x
$$

for any positive integer such that $m_{2} \leq m_{1}$. This with the dominated convergence theorem for the interchange of differentiation and integration, we have from (3.1), (3.2) and (3.3)

$$
\begin{aligned}
& \quad \pi\left|\frac{d^{v}}{d a^{v}} \operatorname{ISE}_{n}(a)-\lim _{n \rightarrow \infty} \frac{d^{v}}{d a^{v}} \operatorname{ISE}_{n}(a)\right| \\
& \leq \mid \int_{-\infty}^{\infty}(-i t)^{v} e^{-i a t} \cdot \varphi_{K}\left(h_{n} t\right)\left(c_{n}(t)-\varphi_{f}(t)\right) \overline{\varphi_{f}(t)} d t \\
& \quad+\int_{-\infty}^{\infty}(-i t)^{v} e^{-i a t}\left(\varphi_{K}\left(h_{n} t\right)-1\right)\left|\varphi_{f}(t)\right|^{2} d t \mid \\
& \leq \sup _{t \in S_{n}}\left|c_{n}(t)-\varphi_{f}(t)\right| \cdot \int_{-\infty}^{\infty}|t|^{v}\left|\varphi_{f}(t)\right| d t+2 \int_{t \in S_{n}}|t|^{v}\left|\varphi_{f}(t)\right| d t \\
& \quad+h_{n} \int_{-\infty}^{\infty}|t|^{v+1}\left|\varphi_{f}(t)\right|^{2} d t \cdot \int_{-\infty}^{\infty}|y K(y)| d y .
\end{aligned}
$$

From Lemma 3.1

$$
\sup _{t \in S_{n}}\left|c_{n}(t)-\varphi_{f}(t)\right|=O\left(T_{n}^{\frac{u-m}{2(u-m+1)}}\right), \text { a.s. }
$$

By the condition (ii), we have

$$
\begin{aligned}
& \int_{t \in S_{n}^{c}}|t|^{v}\left|\varphi_{f}(t)\right| d t \leq T_{n}^{\frac{m-v}{2(u-m+1)}} \int_{t \in S_{n}^{c}}|t|^{m}\left|\varphi_{f}(t)\right| d t \\
& =o\left(T_{n} \frac{\frac{u-v}{2(u-m+1)}}{2(u-m .}\right. \text {, a.s. }
\end{aligned}
$$

Together with (3.7), (3.8) and the order relation of $h_{n}$ and $T_{n}$, we get (3.6).

Note that the right hand side of (3.6) may be written as $O\left(T_{n}^{\frac{u-m}{2(u-m+1)}}+h_{n}\right)$. We can remark about the condition (i) and (ii) of Lemma 3.2 as follows.

Proposmon 3.1. If $\varphi_{f}(t)$ satisfies (2.14) then the condition (i) of Lemma 3.2 holds for any nonnegative integer $m$. And there exists a $\mu>m$ such that (ii) of Lemma 3.2 also holds.

Proof. It is easy to check that

$$
\begin{aligned}
\int_{-\infty}^{\infty}|t|^{m+1}\left|\varphi_{f}(t)\right| d t & \leq \frac{2 A}{\rho^{m+2}} \int_{-\infty}^{\infty} x^{m+1} e^{-x} d x \\
& =\frac{2 A}{\rho^{m+2}}(m+1) ! \\
& <\infty
\end{aligned}
$$

Then we shall show the second assertion of this proposition. Let $\mu_{1}$ be a real positive 
number such that $\mu_{1}>m$. We define the set $V_{n}$ as

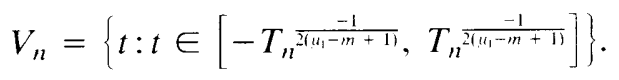

Then we have

$$
\begin{aligned}
& \int_{t \in V_{n}}|t|^{m}\left|\varphi_{f}(t)\right| d t \\
\leq & \frac{2 A}{\rho^{m+1}} \cdot \exp \left(-\rho \cdot T_{n^{2\left(\mu_{1}-m+1\right)}}\right) \cdot \sum_{k=0}^{m} \frac{m !}{k !}\left(\rho \cdot T_{n^{2\left(\mu_{1}-m+1\right)}}\right)^{k} \\
= & o\left(T_{\left.n^{\frac{u-m}{2\left(u_{1}-m+1\right)}}\right)},\right.
\end{aligned}
$$

for any $\mu>m$ by the following fact.

$$
\lim _{x \rightarrow \infty} \frac{x^{\mu}}{e^{x}}=0, \quad \text { for any } \mu \in \boldsymbol{R} .
$$

so if we set $\mu=\mu_{1}$, we get the conclusion.

Lemma 3.3. Under the conditions of Lemma 3.2, we have

$$
\begin{aligned}
& \left|\frac{d^{v}}{d u^{v}} \operatorname{ISE}_{n}(u)\right|_{u=a}-\left.\lim _{n \rightarrow \infty} \frac{d^{v}}{d u^{v}} \operatorname{ISE}_{n}(u)\right|_{u=b} \mid \\
& \leq \frac{|a|+|b|}{\pi} \int_{-\infty}^{\infty}|t|^{v+1}\left|\varphi_{f}(t)\right|^{2} d t+ \begin{cases}O\left(T_{n^{\frac{u}{2(u-m+1}}}\right), & \text { a.s. } \\
O\left(h_{n}\right), & \text { a.s. }\end{cases}
\end{aligned}
$$

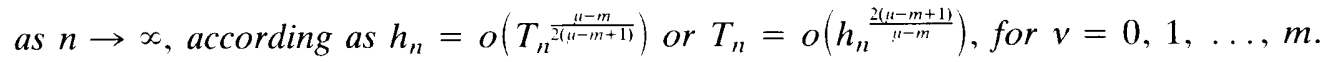

Proof. We may use similar approach to the proof of Lemma 3.2.

$$
\begin{aligned}
& \quad \pi\left|\frac{d^{v}}{d u^{v}} \operatorname{ISE}_{n}(u)\right|_{u=a}-\left.\lim _{n \rightarrow \infty} \frac{d^{v}}{d u^{v}} \operatorname{ISE}_{n}(u)\right|_{u=b} \mid \\
& \leq \sup _{t \in S_{n}}\left|c_{n}(t)-\varphi_{f}(t)\right| \cdot \int_{-\infty}^{\infty}|t|^{v}\left|\varphi_{f}(t)\right| d t+2 \int_{t \in S_{n}}|t|^{v}\left|\varphi_{f}(t)\right| d t \\
& \quad+h_{n} \int_{-\infty}^{\infty}|t|^{v+1}\left|\varphi_{f}(t)\right|^{2} d t \cdot \int_{-\infty}^{\infty}|y K(y)| d y \\
& \quad+\int_{-\infty}^{\infty}|t|^{v}\left|e^{-i a t}-e^{-i b t}\right|\left|\varphi_{f}(t)\right|^{2} d t \\
& \leq(|a|+|b|) \int_{-\infty}^{\infty}|t|^{v+1}\left|\varphi_{f}(t)\right|^{2} d t+O\left(T_{n^{\frac{u-m}{2(u-m+1)}}}\right), \text { a.s. }
\end{aligned}
$$

if $h_{n}=o\left(T_{n^{\frac{u-m}{2(u-m+1)}}}\right)$, and otherwise is clear. Hence we get the conclusion.

Now we can state our main theorems.

THEOREM 3.1. If the conditions of Lemma 3.2 are satisfied for $m=2$, then we have 


$$
\begin{aligned}
& a_{n}=O\left(T_{n^{\frac{1}{4}}}^{\frac{1}{4}\left(1-\frac{1}{\mu-1}\right)}\right) \text {, a.s. for } h_{n}=o\left(T_{n^{\frac{u-2}{2(u-1)}}}\right) \\
& =O\left(h_{n}^{1 / 2}\right), \quad \text { a.s. for } T_{n}=o\left(h_{n}^{\left.\frac{2(u-1)}{u-2}\right)}\right.
\end{aligned}
$$

for sufficiently large $n$.

Proof. We shall only show for the case $h_{n}=o\left(T_{\left.n^{\frac{u-2}{2(u-1)}}\right)}\right)$ as the otherwise can be shown in the same way. By expanding $\frac{d}{d u} \operatorname{ISE}_{n}(u)$ and $\lim _{n \rightarrow \infty} \frac{d}{d u} \operatorname{ISE}_{n}(u)$ about $u=0$, we have

$$
\begin{aligned}
& \left.\frac{d}{d u} \operatorname{ISE}_{n}(u)\right|_{u=a}-\left.\lim _{n \rightarrow \infty} \frac{d}{d u} \operatorname{ISE}_{n}(u)\right|_{u=a}-\left[\left.\frac{d}{d u} \operatorname{ISE}_{n}(u)\right|_{u=0}-\left.\lim _{n \rightarrow \infty} \frac{d}{d u} \operatorname{ISE}_{n}(u)\right|_{u=0}\right] \\
& =a \cdot\left[\left.\frac{d^{2}}{d u^{2}} \operatorname{ISE}_{n}(u)\right|_{u=\theta a}-\left.\frac{d}{d u}\left(\lim _{n \rightarrow \infty} \frac{d}{d u} \operatorname{ISE}_{n}(u)\right)\right|_{u=\theta^{\prime} a}\right]
\end{aligned}
$$

where $\theta, \theta^{\prime} \in(0,1)$. It is obvious that

$$
\frac{d^{v-1}}{d u^{v-1}}\left(\lim _{n \rightarrow \infty} \frac{d}{d u} \operatorname{ISE}_{n}(u)\right)=\lim _{n \rightarrow \infty} \frac{d^{v}}{d u^{v}} \operatorname{ISE}_{n}(u)
$$

for any positive integer $v \leq m$. And so by Lemma 3.3, we have

$$
\begin{aligned}
& \left.\frac{d^{2}}{d u^{2}} \operatorname{ISE}_{n}(u)\right|_{u=\theta_{n} a_{n}}-\left.\frac{d}{d u}\left(\lim _{n \rightarrow \infty} \frac{d}{d u} \operatorname{ISE}_{n}(u)\right)\right|_{u=\theta^{\prime}{ }_{n} a_{n}} \\
\leq & \frac{1}{\pi}\left(\left|\theta_{n}\right|+\left|\theta_{n}^{\prime}\right|\right)\left|a_{n}\right| \int_{-\infty}^{\infty}|t|^{3}\left|\varphi_{f}(t)\right|^{2} d t+O\left(T_{n^{\frac{u-2}{2(u-1)}}}\right) \\
= & O\left(a_{n}+T_{\left.n^{\frac{u-2}{2(u-1)}}\right)}\right), \text { a.s. }
\end{aligned}
$$

since $\theta_{n}, \theta^{\prime}{ }_{n} \in(0,1)$. Therefore we can rewrite $(3.10)$ as

$$
O\left(T_{n^{\frac{u-2}{2(u-1)}}}^{2(u)}=a_{n} \cdot O\left(a_{n}+T_{n^{\frac{u-2}{2(u-1)}}}\right),\right. \text { a.s. }
$$

by Lemma 3.2. Hence we have (3.9).

It is well known that the asymptotically optimal convergence order of the window width $h_{n}$ is $n^{-1 / 5}$, i.e. $n^{1 / 5} h_{n} \rightarrow$ const. as $n \rightarrow \infty$, see Prakasa Rao [14]. In this case it is easy to check if $2<\mu \leq \frac{8}{3}$ then $h_{n}=o\left(T_{n^{\frac{\mu-2}{2(\mu-1)}}}\right)$, and if $\mu \geq \frac{8}{3}+0.1$ then we have $T_{n}=o\left(h_{\left.n^{\frac{2(u-1)}{u-2}}\right)}\right.$.

THEOREM 3.2. Under the conditions of Lemma 3.2 for $m=2$, we have

$$
\begin{aligned}
\operatorname{DISE}_{n}\left(a_{n}\right) & =O\left(T_{n^{\frac{1}{3}}\left(1-\frac{1}{u-1}\right)}\right), \text { a.s. for } h_{n}=o\left(T_{\left.n^{\frac{u-2}{2(u-1)}}\right)}\right. \\
& =O\left(h_{n}^{1 / 2}\right), \quad \text { a.s. for } T_{n}=o\left(h_{n}^{\frac{2(u-1)}{u-2}}\right)
\end{aligned}
$$

for sufficiently large $n$. 
Proof. We shall also show for $h_{n}=o\left(T_{n^{\frac{u-2}{2(u-1)}}}\right)$ case. Since from the proof of Lemma 3.2

$$
\begin{aligned}
& \left.\frac{1}{\pi}\left|\pi \cdot \operatorname{DISE}_{n}(a)-\int_{-\infty}^{\infty}\{1-\cos (a t)\}\right| \varphi_{f}(t)\right|^{2} d t \mid \\
\leq & 2 \sup _{t \in S_{n}}\left|c_{n}(t)-\varphi_{f}(t)\right| \cdot \int_{-\infty}^{\infty}\left|\varphi_{f}(t)\right| d t+4 \int_{t \in S_{n}}\left|\varphi_{f}(t)\right| d t \\
& +2 h_{n} \int_{-\infty}^{\infty}|t|\left|\varphi_{f}(t)\right|^{2} d t \cdot \int_{-\infty}^{\infty}|y K(y)| d y . \\
= & O\left(T_{n^{\frac{u-2}{2(u-1)}}}\right), \text { a.s. }
\end{aligned}
$$

therefore we have (3.12) which is more accurate than (2.16)

$$
\operatorname{DISE}_{n}(a)=\frac{1}{\pi} \int_{-\infty}^{\infty}\{1-\cos (a t)\}\left|\varphi_{f}(t)\right|^{2} d t+O\left(T_{n^{\frac{u-2}{2(u-1)}}}\right), \text { a.s. }
$$

uniformly for $a \in \boldsymbol{R}$. By Theorem 3.1

$$
\begin{aligned}
\int_{-\infty}^{\infty}\left\{1-\cos \left(a_{n} t\right)\right\}\left|\varphi_{f}(t)\right|^{2} d t & \leq\left|a_{n}\right| \int_{-\infty}^{\infty}|t|\left|\varphi_{f}(t)\right|^{2} d t \\
& =O\left(T_{n^{\frac{1}{4}}\left(1-\frac{1}{u-1}\right)}\right), \text { a.s. }
\end{aligned}
$$

Thus we get the conclusion.

Let $\hat{a}_{n}$ be any estimator of the $a_{n}$ then by the definition of the $a_{n}$, we have

$$
\operatorname{DISE}_{n}\left(a_{n}\right) \leq \operatorname{DISE}_{n}\left(\hat{a}_{n}\right) \text {, a.s. }
$$

for all $n \in N$. And also we have

$$
\operatorname{DISE}_{n}\left(a_{n}\right) \leq 0 \text {, a.s. }
$$

for all $n \in N$. We define the estimator $\hat{a}_{n}$ is asymptotically efficient if $n \geq N$ then $\operatorname{DISE}_{n}\left(\hat{a}_{n}\right) \leq 0$ for some $N>0$, with probability one. The asymptotic property of $\operatorname{ISE}_{n}(0)$ has been studied by Hall [8], it is obvious that the order of convergence of $\operatorname{ISE}_{n}\left(a_{n}\right)$ is the same as $\operatorname{ISE}_{n}(0)$. So we can say $\operatorname{DISE}_{n}\left(\hat{a}_{n}\right)$ is desired that it converges to 0 as slower as could. Theorem 3.2 gives the lower bound of the convergence discussed above. Namely, if the estimator $\hat{a}_{n}$ is asymptotically efficient then $\operatorname{DISE}_{n}\left(\hat{a}_{n}\right)$ can not converge to 0 slower than the order $O\left(T_{n}^{\frac{1}{(1)}\left(1-\frac{1}{\mu-1}\right)}\right)$ or $O\left(h_{n}^{1 / 2}\right)$ with probability one.

\section{Simulation Study}

In this section we shall show the examples for the following case.

We have,

$$
\begin{aligned}
& f(x)=\frac{1}{\sqrt{2 \pi}} \exp \left(-\frac{1}{2} x^{2}\right) \\
& K(y)=\frac{1}{\sqrt{2 \pi}} \exp \left(-\frac{1}{2} y^{2}\right)
\end{aligned}
$$




$$
\begin{gathered}
\operatorname{ISE}_{n}\left(a, h_{n}\right)=\frac{1}{2 \sqrt{\pi} n h_{n}}\left[1+\frac{2}{n} \sum_{j<l}^{n} \exp \left\{-\frac{1}{4}\left(\frac{\mathrm{X}_{\mathrm{j}}-X_{l}}{h_{n}}\right)^{2}\right\}\right] \\
-\frac{1}{n}\left(\frac{2}{\pi\left(1+h_{n}^{2}\right)}\right)^{\frac{1}{2}} \cdot \sum_{j=1}^{n} \exp \left\{-\frac{\left(X_{j}-a\right)^{2}}{2\left(1+h_{n}^{2}\right)}\right\}+\frac{1}{2 \sqrt{\pi}} .
\end{gathered}
$$

We define $\operatorname{DISE}_{n}\left(a, h_{n}^{*}\right)$ as

$$
\operatorname{DISE}_{n}\left(a, h_{n}^{*}\right)=\operatorname{ISE}_{n}\left(a, h_{n}^{*}\right)-\operatorname{ISE}_{n}\left(0, h_{n}^{*}\right),
$$

where $h_{n}^{*}$ is given by

$$
\operatorname{ISE}_{n}\left(0, h_{n}^{*}\right)=\min _{0<h_{n}<x} \operatorname{ISE}_{n}\left(0, h_{n}\right) .
$$

Secondly, we conduct a simulation to estimate

$$
E\left[\frac{\min _{-x<a<x} \operatorname{ISE}_{n}\left(a, h_{n}^{*}\right)}{\operatorname{ISE}_{n}\left(0, h_{n}^{*}\right)}\right],
$$

by calculating

$$
\frac{1}{N} \sum_{s=1}^{N} \frac{\min _{-x<a<x} \operatorname{ISE}_{n}\left(a, h_{n}^{*}\right)}{\operatorname{ISE}_{n}\left(0, h_{n}^{*}\right)}
$$

for sample size $n=50(N=100)$ and $n=100(N=50)$. (4.1) may be defined as a relative efficiency of the estimation with respect to the location shift r.v. $a_{n}$. As a result we have $58 \%$ for $n=50$, and $62 \%$ for $n=100$ respectively. These percentages motivate us to construct an estimator of the $a_{n}$.

The author has been considering the estimator of $a_{n}$. For example, it may be worth while to study the following two types of the estimators.

I. From (2.19) we may construct a natural estimator,

$$
\hat{a}_{n}=\frac{-\sum_{j=1}^{n} \widehat{\Psi}_{n}\left(X_{j}\right)}{\left.\sum_{j=1}^{n} \frac{d}{d u} \widehat{\Psi}_{n}\left(x_{j}-u\right)\right|_{\mathrm{u}=0}} .
$$

where $\widehat{\Psi}_{n}(X)$ is given by

$$
\widehat{\Psi}_{\mathrm{n}}(X)=\operatorname{Im}\left[\int_{-\infty}^{\infty} t e^{i X t} \cdot \varphi_{K}\left(h_{n} t\right) \cdot \overline{\widehat{\varphi}_{f}(t)} d t\right],
$$

and $\widehat{\varphi}_{f}(t)$ is an estimator of the characteristic function $\widehat{\varphi}_{f}(t)$.

II. The corss-validation method. Define the equation as

$$
C V(h, a)=\int_{-\infty}^{\infty} f_{n}^{2}(x) d x-\frac{2}{n} \sum_{j=1}^{n} f_{n-1,-j}\left(X_{j}-a\right),
$$

where

$$
f_{n-1,-j}(x)=\frac{1}{(n-1) h_{n}} \sum_{i \neq j}^{n} K\left(\frac{x-X_{i}}{h_{n}}\right)
$$


And find the $\left(\widehat{h}_{n}, \hat{a}_{n}\right)$ such that

$$
C V\left(\widehat{h}_{n}, \hat{a}_{n}\right)=\min _{-x<a<x} \min _{h>0} C V(h, a) .
$$

The cross-validation method to find the asymptotically optimal window width $h_{n}$ has been studied by many authors, see, for example Bowman [3] and Rudemo [15].

\section{Acknowledgement}

The author would like to express his thanks to Prof. Y. Washio of Keio University for his encouragement and many helpful advices. He also appreciates the members of his seminar, and Prof. M. Sibuya and Prof. R. Shibata of Keio University for their critical comments and suggestions. In addition, he appreciates the reviewer's comments.

\section{References}

[1] Billingsley, P.: Convergence of Probability Measures, Wiley, New York, (1968).

[2] Blackman. J.: On the approximation of a distribution function by an empiric distribution, Ann. Math. Statist. 26, (1955), 256-267.

[3] Bowman, A.: An alternative method of cross-validation for the smoothing of density estimates, Biometrika 71, (1984), 353-360.

[4] Chung, K. L.: An estimate concerning the kolmogoroff limit distribution. Trans. Amer. Math. Soc. 67, (1949), 36-56.

[5] Csörgö, S.: Limit behaviour of the empirical characteristic function, Ann. Prob. 9, (1981), 130-140.

[6] Feunrverger, A. and Mureika, R. A.: The empirical characteristic function and its applications, Ann. Statist. 5, (1977), 88-97.

[7] HALl, P.: Laws of iterated logarithm for nonparametric density estimators, Zeit. Wahrscheinl.-theoric 56. (1981), 47-61.

[8] HALL, P.: Limit theorems for stochastic measures of the accuracy of density estimators, Stoch. Processes Appl. 13, (1982), 11-25.

[9] Härdle, W.: Applied Nonparametric Regression, Cambridge Univ. Press, (1990).

[10] Heатнсоте, C. R.: The integrated squared error estimation of parameters, Biometrika 64, (1977), 255264.

[11] Huber, P. J.: Robust estimation of a location shift parameter, Ann. Math. Statist. 35. (1964), 73-101.

[12] Izenman, A. J.: Recent developments in nonparametric density estimation, J. Amer. Statist. Ass. 86, (1991), 205-224.

[13] Lukıcs, E.: Characteristic Functions, 2nd. ed. Griffin, London, (1970).

[14] Marcus, M. B.: Weak convergence of the empirical characteristic function, Ann. Prob. 9, (1981), 194201.

[15] Prakasa Rao, B. L. S.: Nonparametric Fuctional Estimation, Academic Press. (1983).

[16] Rudemo, M.: Empirical choice of histograms and kernel density estimators, Scand. J. Statist. 9, (1982), $65-78$.

[17] Scotr, D. W.: Averaged shifted histograms: effective nonparametric density estimators in several dimensions, Ann. Statist. 13, (1985), 1024-1040.

[18] Serfling, R. J.: Approximation Theorems of Mathematical Statistics, Wiley, New York, (1980)

[19] Watson, G. R. and Leadbetter, M. R.: On the estimation of the probability density, I, Ann. Math. Statist. 34, (1963), 480-491. 
Received May 13, 1992

Revised September 24, 1992

Communicated by $T$. Yanagawa 